\title{
Stability of blueberry polyphenols subjected to in vitro digestion with human gastrointestinal enzymes
}

\author{
Nikolina Liović ${ }^{1}$, Tea Bilušić ${ }^{2}$, Anet Režek Jambrak ${ }^{3}$, Greta Krešić ${ }^{1}$ and Joško Markić ${ }^{4}$ \\ ${ }^{1}$ Faculty of Tourism and Hospitality Management, University of Rijeka, Opatija, Croatia, \\ ${ }^{2}$ Faculty of Chemistry and Technology, University of Split, Split, Croatia, \\ ${ }^{3}$ Faculty of Food Technology and Biotechnology, University of Zagreb, Zagreb, Croatia and \\ ${ }^{4}$ University Hospital Split, Split, Croatia
}

\section{Abstract}

Blueberries (Vaccinium corymbosum, L.) are a valuable source of health-promoting bioactive compounds, particularly polyphenols. The beneficial effects of polyphenols on health depend not only on food sources but also on their stability, which can vary depending on the post-harvest processing methods, the matrix in which they are incorporated, and endogenous factors such as microbiota and digestive enzymes. The aim of this study was to investigate the influence of high-intensity ultrasound treatment on in vitro gastrointestinal stability of the total polyphenol content and antioxidant capacity of blueberries. Blueberry puree was treated with high-intensity ultrasound using different amplitudes $(30,45$ and $60 \mu \mathrm{m})$ and treatment times (3, 6 and 9 min), and a part of sample was used as a control (untreated sample). Total phenolic content (TPC) and antioxidant capacity (by DPPH and FRAP methods) were determined prior to and after two-phase in vitro digestion process (gastric and duodenal) using human gastrointestinal enzymes. The results showed a high stability of total blueberry polyphenols and antioxidant activity during simulated gastric digestion step, both in untreated and ultrasound treated samples. The use of high-intensity ultrasound had a significant effect on the stability of polyphenols and their antioxidant activity after simulated digestion in duodenum. Significant increase in polyphenol content after duodenal digestion step was determined in three ultrasound treated samples with longer treatment time (6 and 9 minutes), and only sample with significant reduction of TPC was untreated sample. Modification of the food matrix as a result of cavitation from ultrasound treatment may be the reason for higher stability of polyphenol during in vitro digestion. After in vitro digestion blueberry polyphenols still possessed antioxidant activity that may be directly related to their beneficial effects on health. The highest antioxidant activity measured with DPPH and FRAP method was determined for sample treated with maximum amplitude $(60 \mu \mathrm{m})$ and longest time $(9$ minutes). Determination of the stability of phenolic compounds is important as it affects their bioavailability for uptake as well as their possible beneficial effects on intestinal epithelial tissue such as reduction of oxidative stress and cancer prevention. Our results showed that ultrasound treatment can preserve the blueberry phenolics and their antioxidant characteristics after in vitro digestion.

\section{Conflict of Interest}

There is no conflict of interest 